\title{
Mobility of Hematite Submicron Particles in Water Solutions of Sugar
}

\author{
P. FORNAL ${ }^{a}$ AND J. STANEK ${ }^{b}$ \\ ${ }^{a}$ Institute of Physics, Cracow University of Technology \\ Podchorążych 1, 30-083 Kraków, Poland \\ ${ }^{b}$ Marian Smoluchowski Institute of Physics, Jagiellonian University \\ Reymonta 4, 30-059 Kraków, Poland
}

\begin{abstract}
The mobility of the $100 \mathrm{~nm} \mathrm{Fe}_{2} \mathrm{O}_{3}$ particles in dense water solutions of sugar (sucrose) was determined from the analysis of the resonance absorption line shape of the Mössbauer spectra recorded in the $-5^{\circ} \mathrm{C}$ to $40^{\circ} \mathrm{C}$ temperature range for different sugar concentrations. The discrepancy between the experimental data and the prediction of the classical theory of Brownian movement are interpreted in the term of the short observation time and the interaction between the solid particles in fluids, which extends in water up to $300 \mathrm{~nm}$. The sedimentation process in the studied colloids was observed.
\end{abstract}

PACS numbers: 05.40.Jc, 47.57.ef, 47.57.J-, 47.61.Cb

\section{Introduction}

The rapidly expanding nano-biotechnology causes an increased interest in the field of the mobility of submicron particles in viscous organic media. The nanoparticles and microspheres are considered as promising drugs and antigen delivery systems; the encapsulation of the medicaments may lead to the release of them in a controlled way for the long time period, protecting from degradation. In addition, such particles may be treated as local probes for the three-dimensional mesoscopic systems. The interaction of submicron particles in colloids is a hot topic in the classical colloid technology, such as paint or food production.

The opportunity of the Mössbauer spectroscopy in the study of the truly microscopic, one-dimensional movement of the absorbing particles is well known. The modification of the Mössbauer line shape was described by Singwi and Sjőlander [1] as the time and space Fourier transform of the time dependent self-correlation function, $G(r, t)$, of the absorbing objects 


$$
\sigma(\omega)=\left(\sigma_{0} / 4 \tau\right) \iint \exp \left(\mathrm{i}\left(k \cdot r-\left(\omega-\omega_{0}\right) t\right)-t / 2 \tau\right) G(r, t) \mathrm{d} r \mathrm{~d} t,
$$

where $\sigma_{0}$ is the resonant cross-section and $\tau$ is the mean lifetime of the excited state (here $141 \mathrm{~ns}$, for the case of ${ }^{57} \mathrm{Fe}$ ). The $G(r, t)$ for the free particles was proposed by Bhide et al. [2] in a form of the Langevin distribution function, while Nowik et al. [3,4] described the dynamics of the iron bearing biomolecules in terms of the overdamped oscillator.

The microscopic bases of the mobility of the submicron particles in liquids are Brownian movements. Within the classical approach the distribution of their one-dimensional velocity is Gaussian with standard deviation

$$
\sigma_{v}=(2 D / \tau)^{1 / 2}
$$

where $D$ is self-diffusion coefficient,

$$
D=k T / 6 \pi \eta r,
$$

where $r$ is radius of the particles and $\eta$ is the viscosity of the medium. The corresponding broadening $\Delta \Gamma$ of resonant absorption line in the case of ${ }^{57} \mathrm{Fe}$ is

$$
\Delta \Gamma[\mathrm{mm} / \mathrm{s}]=1.44 \times 10^{14} \mathrm{D}
$$

or

$$
\Delta \Gamma[\mathrm{mm} / \mathrm{s}]=1.1 \times 10^{-7} \mathrm{~T} / \eta r .
$$

For $T=300 \mathrm{~K}, r=100 \mathrm{~nm}$ and $\eta=100 \mathrm{cP}=0.1 \mathrm{~Pa}$ s the broadening $\Delta \Gamma=3.2 \mathrm{~mm} / \mathrm{s}$ is expected. The given above Eq. (2a) for $\sigma_{v}$ is valid in the case, where the observation time, here $\tau$, is much longer than the relaxation time, $t_{\mathrm{R}}$, i.e. the time when the moving particles come to the rest

$t_{\mathrm{R}}=m / 6 \pi \eta r$.

For hematite particles with $r=10^{-6} \mathrm{~m}, m=2.2 \times 10^{-14} \mathrm{~kg}$. In liquid with $\eta=0.001 \mathrm{~N} \mathrm{~s} \mathrm{~m}^{-2}(1 \mathrm{cP})$ the relaxation time is $t_{\mathrm{R}}=1.2 \times 10^{-5} \mathrm{~s}$, thus the condition $t_{\mathrm{R}} \ll \tau$ is fulfilled in the classical experiments, for which $\tau$ is in the range of seconds. In the case of ${ }^{57} \mathrm{Fe}$ Mössbauer spectroscopy $t_{\mathrm{R}}$ may be of the order of $\tau=141 \mathrm{~ns}$ and the classical approximation may be not valid.

The long term aim of this work is to develop the methodology for the study of mobility of iron bearing submicron particles in viscous media, based on the analysis of the precisely determined shape of the Mössbauer line. The main problems are: (a) what is the shape of the resonant line in the case of Brownian movement, (b) how is the relation between the line broadening and the mobility of the particles, (c) what is the interaction between particles, (c) what is the interaction between particles and surfaces, (d) what is the relation between microscopic and macroscopic viscosity, and (e) may the submicron particles be used as probes of the soft matter mesoscopic structures as for example cellular amine gels [5].

In this work we describe the results of the Mössbauer study of the hematite submicron particles in concentrated water solution of sugar (sucrose, $\mathrm{C}_{12} \mathrm{H}_{22} \mathrm{O}_{11}$ ). The concentration and temperature dependence of the viscosity of such solution is known, thus we may attempt to describe the evolution of the shape of the 
Mössbauer as the function of these macroscopic parameters. Moreover, the effect of the sedimentation of the particles was observed as the increase in the narrow line fraction in the spectrum. Finally, the effect of the particle-particle interactions was estimated by changing the concentration of the hematite particles in water.

\section{Experimental}

\subsection{Samples}

The $\mathrm{Fe}_{2} \mathrm{O}_{3}$ particles, 100(10) nm in diameter, used as a commercial pigment (MIXOL 4), were distributed in the water sugar solution with 40, 60, and 80 wt.\% concentration of sugar. The liquids were put in sealed sample holders, $6 \mathrm{~mm}$ thick. In the case of colloids in water, the hematite concentration varied between $6 \%$ wt. and $70 \%$ wt., which corresponds to the average nearest particle-particle distance from $370 \mathrm{~nm}$ to $130 \mathrm{~nm}$; measured from the particles' centers. The distribution of the diameter of the particles, and their fairly spherical shape were determined using scanning electron microscope.

\subsection{Measurements}

The ${ }^{57} \mathrm{Fe}$ Mössbauer spectra were recorded in the $-5^{\circ} \mathrm{C}$ to $40^{\circ} \mathrm{C}$ temperature range in the vertical transmission geometry. Due to the complicated spectral shape, broad lines and small effect of the resonant absorption, the statistics up to about $10^{7}$ in each 1024 channel were required. The application of fast amplifiers made possible reaching the counting rate of $2 \times 10^{4}$ counts/s in the Mössbauer line. The hyperfine parameters of the used powder of $\mathrm{Fe}_{2} \mathrm{O}_{3}$ were precisely determined between $-10^{\circ} \mathrm{C}$ and $40^{\circ} \mathrm{C}$.
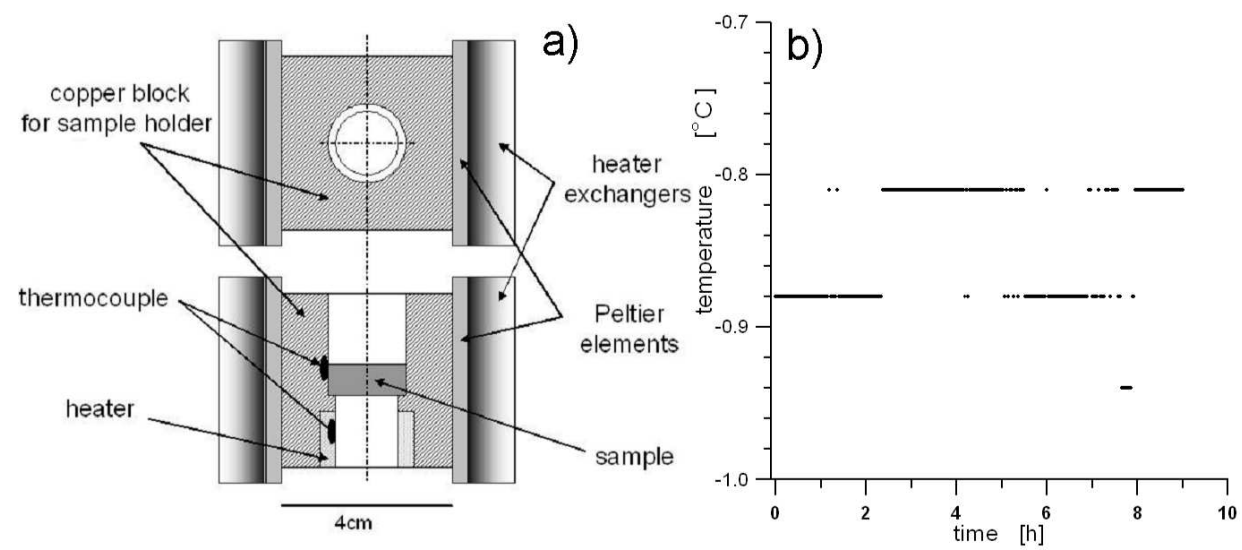

Fig. 1. (a) The scheme of the Mössbauer cryostat for the biomedical applications. The free space is filled by styrofoam. (b) Time record of the temperature stabilization during the experiment.

The samples were placed in the home-made cryostat, based on the Peltier elements. This cryostat, dedicated for biomedical studies at temperatures close 
to room temperature, exhibits perfect temperature stabilization $\left(0.1^{\circ} \mathrm{C}\right)$ and its compact geometry allows measurements with high count rates. The scheme of the cryostat is shown in Fig. 1.

\section{Results}

The examples of the spectra of the hematite particles in water solutions of sugar are shown in Fig. 2. The spectra were fitted with sets (up to 5) of sextets

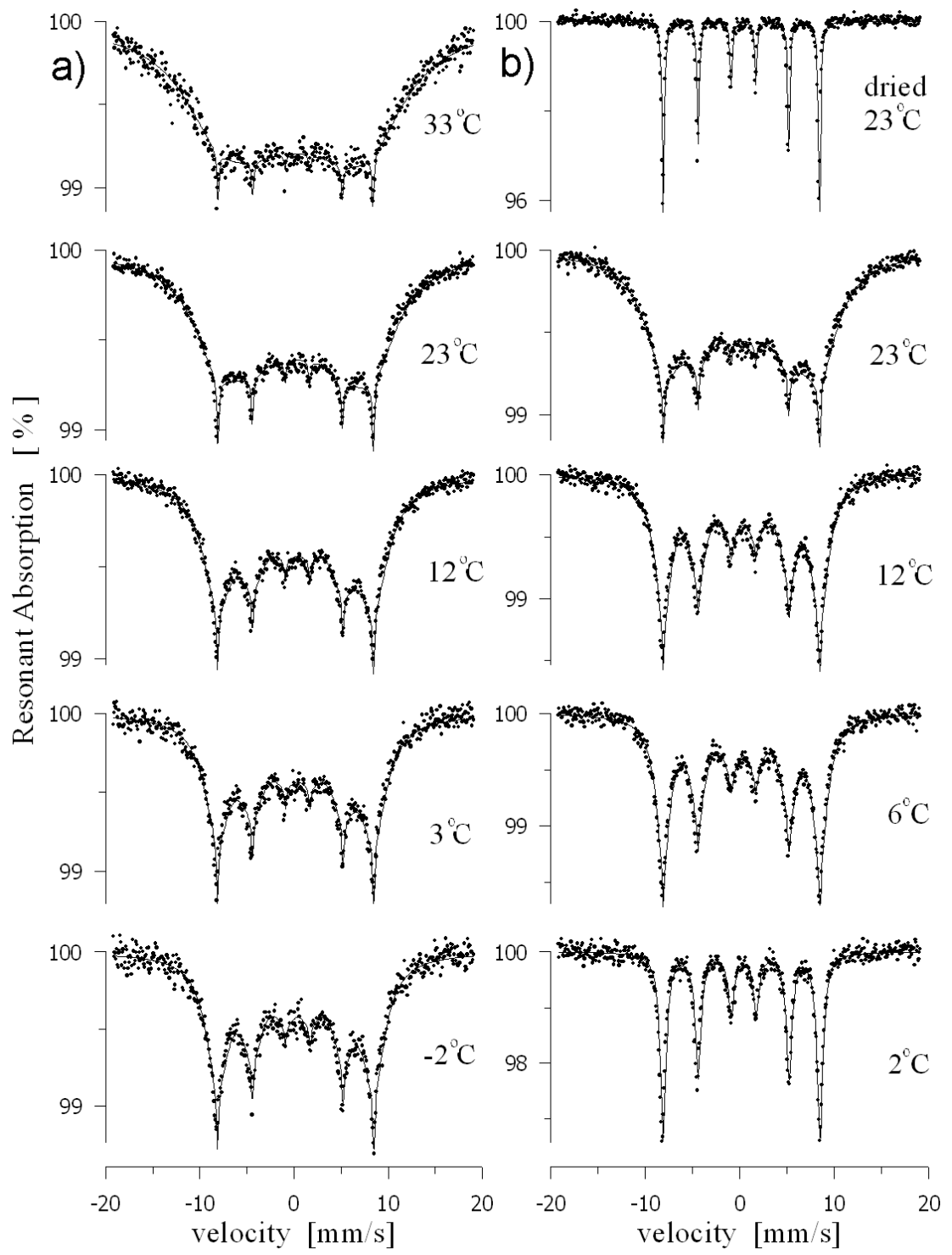

Fig. 2. The ${ }^{57} \mathrm{Fe}$ Mössbauer spectra of the $100 \mathrm{~nm} \mathrm{Fe}{ }_{2} \mathrm{O}_{3}$ particles in $60 \%$ wt. (a) and $80 \%$ wt. sugar solutions (b) at temperatures as marked. 
with hyperfine parameters constrained to these of bulk hematite, measured at corresponding temperatures. Thus, the possible, in principle, phase transitions in the studied small particles of hematite become irrelevant. Finally, only the intensity and the line width (common for all lines in the sextet) were fitted and the line profile for each spectrum has been reconstructed. Due to the significant broadening of the lines, the amplitude of the resonant absorption was small and the samples may be treated as thin samples.

The temperature dependence of the broadening of the Mössbauer line is obvious from the visual inspection of the spectra. The temperature influences the particle mobility mainly through the variation of the viscosity. For example, the viscosity of $60 \%$ wt. sugar solution in water varies from $0.24 \mathrm{~Pa} \mathrm{~s}$ at $0^{\circ} \mathrm{C}$ to $0.021 \mathrm{~Pa} \mathrm{~s}$ at $40^{\circ} \mathrm{C}$. The dependence of the "effective broadening", $\Delta \Gamma=\left(\Sigma f_{i} \Delta \Gamma_{i}^{2}\right)^{1 / 2}$, where $f_{i}$ and $\Delta \Gamma_{i}$ are the fractions and the broadening of the subspectra lines, on the viscosity of the $60 \%$ wt. sugar solution is displayed in Fig. 3. Here, the broadenings were normalized to the $300 \mathrm{~K}$ temperature by dividing their experimental values by $T / 300$, where $T$ was the temperature of the experiment. The narrow line fraction, with line width of $0.28 \mathrm{~mm} / \mathrm{s}$, comparable to that for powder sample, was assigned to the sediment of the hematite. This fraction with area of $2-5 \%$ of the spectrum, increasing with the time of subsequent measurements, was excluded from the analysis. The increase in the narrow line contribution proves that the systems, at least for the less concentrated solutions, are not fully stable.

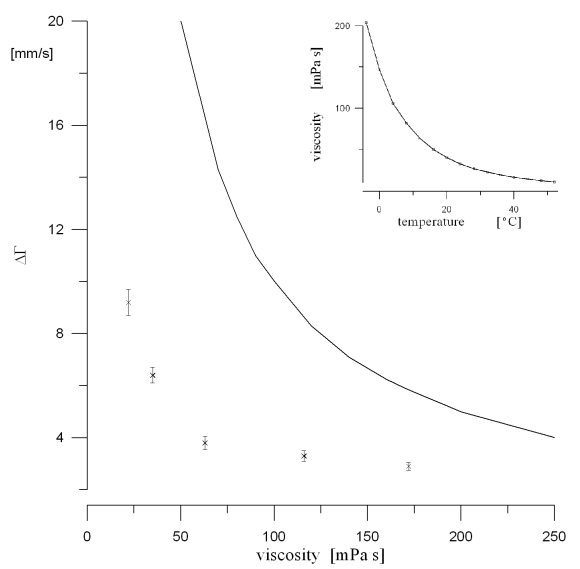

Fig. 3. The broadening of the absorption line of ${ }^{57} \mathrm{Fe}$ Mössbauer spectra of the $\mathrm{Fe}_{2} \mathrm{O}_{3}$ particles in $60 \%$ wt. sugar solutions in water vs. viscosity of the solution. The viscosity was alternated by the temperature; the broadening is normalized to the $300 \mathrm{~K}$ temperature, see text. Solid line: theory, points: experiment. In the inset the temperature dependence of the viscosity of the $60 \%$ wt. sugar solution is shown.

Finally, the influence of the concentration of the hematite particles in water was studied. Knowing the mass, density, and size of particles their number in the 


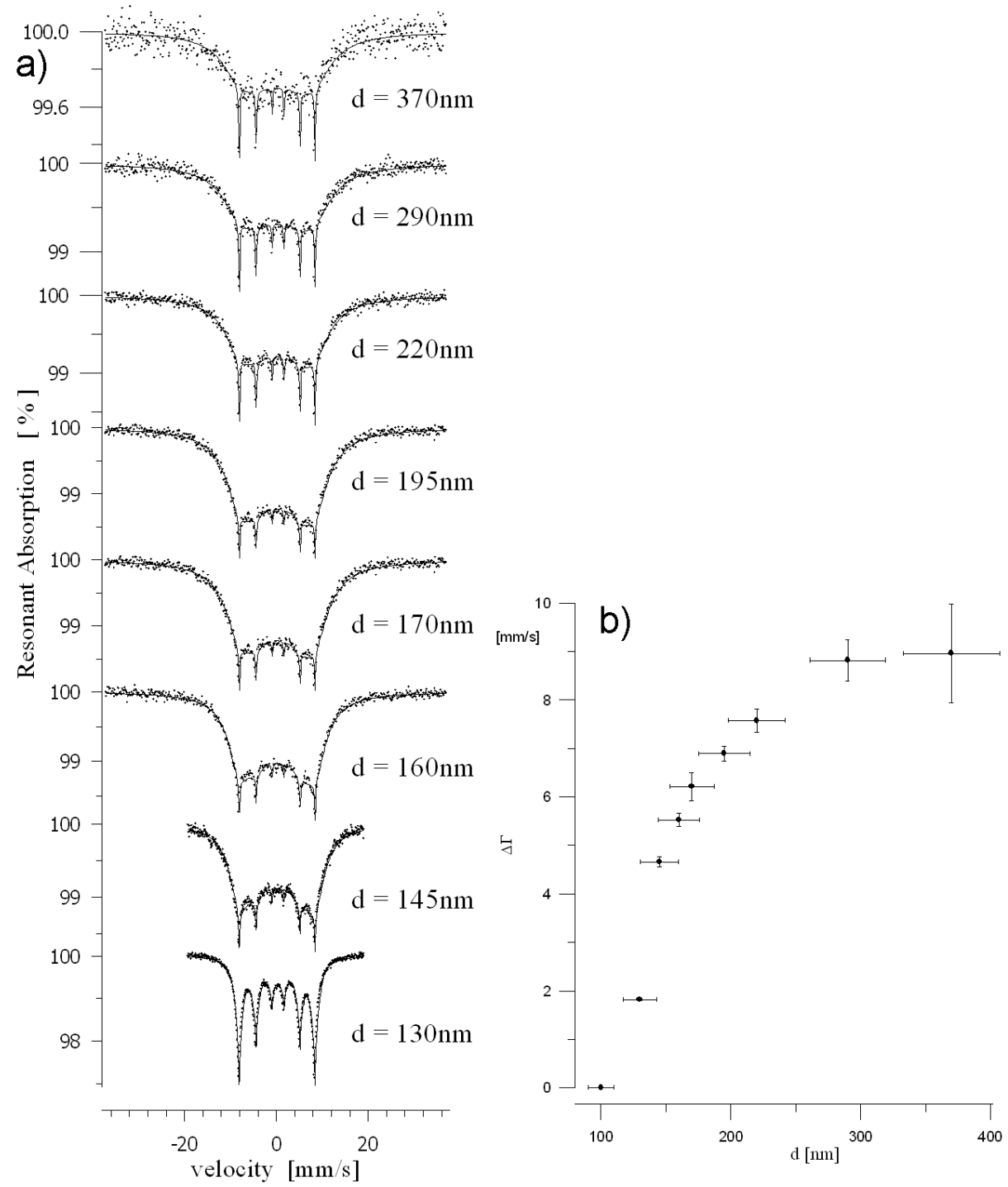

Fig. 4. (a) The ${ }^{57} \mathrm{Fe}$ Mössbauer spectra of $100 \mathrm{~nm} \mathrm{Fe}_{2} \mathrm{O}_{3}$ particles in water recorded for different average nearest particle-particle distances, controlled by the concentration of the particles. (b) The corresponding line broadening of the ${ }^{57} \mathrm{Fe}$ Mössbauer spectra.

volume unit of the solution was calculated. Next, the average nearest particleparticle distance was estimated. In Fig. 4, there are shown the spectra of hematite with different nearest particle-particle distances in water and the corresponding broadening of the line width. It may be assumed that for distance $400 \mathrm{~nm}$ (i.e. $300 \mathrm{~nm}$ from surface to surface) the mobility of the particles is stable. 


\section{Discussion}

Calculated, according to the "classical" Eqs. (2a)-(2c) broadenings of the Mössbauer absorption lines are compared with the experimental data in Fig. 4 for $60 \%$ wt. solution of sugar, showing a significant discrepancy. It means that the dynamic processes are quite complicated. Some hints for the interpretation of that result come from the observed increase in the mobility of the particles with decreasing their concentration in water, i.e. with the increase in their average distances in the liquid. Corresponding spectra and the increase in the line broadening with the progressing dilution is shown in Fig. 4. This is a convincing evidence that particles interact through the liquid. The critical distance for these interactions in water is $300 \mathrm{~nm}, 3$ times bigger than the diameter of the particles. This effect certainly calls for advanced mathematical evaluations. At the present stage we may describe it as an adhesion of water molecules to the hematite particles which increase their "effective" mass and radius, even 4 times. This certainly slows down the mobility, cf. Eqs. (2a)-(2d), and may qualitatively explain the discrepancy displayed in Fig. 3. In addition, the increase in the mass of the particles enhances the relaxation time $t_{\mathrm{R}}$, cf. Eq. (3), which now may be longer than the " $57 \mathrm{Fe}$ Mössbauer observation time" of $141 \mathrm{~ns}$ and the applicability of Eqs. (2a) $-(2 \mathrm{~d})$ becomes questionable. Nevertheless, even at the present stage this result opens new research perspectives.

The growing with the time of the narrow line fraction is an evidence of the sedimentation of the powder. Thus, to avoid this problem, in the further experiments the horizontal geometry, with the bottom of the sample holder out of the gamma beam, will be considered.

\section{Conclusion}

The presented results of the rather simple experiments demonstrate the potential application of the Mössbauer spectroscopy in a "forgotten" by this technique field of research. It is obvious that the submicron iron bearing particles may be used as the local, in a mesoscopic scale, probes of the soft matter. The rather qualitative, so far, observations are the starting point for the further, more sophisticated experiments described by advanced theoretical models and effective numerical methods.

\section{References}

[1] K.S. Singwi, A. Sjőlander, Phys. Rev. 120, 1093 (1960).

[2] G. Bhide, G. Sundaram, H. Bhasin, T. Bonchev, Phys. Rev. B 3, 673 (1971).

[3] I. Nowik, S.G. Cohen, E.R. Bauminger, S. Ofer, Phys. Rev. Lett. 50, 1528 (1983).

[4] I. Nowik, E.R. Bauminger, S.G. Cohen, S. Ofer, Phys. Rev. A 31, 2291 (1985).

[5] P. Fornal, J. Stanek, A. Wilk, Hyperfine Interact., submitted for publication. 